\title{
THE PROBLEM OF EIGENVALUES IN SOME SINGULAR HOMOGENEOUS VOLTERRA INTEGRAL EQUATIONS
}

\author{
LL. G. CHAMBERS
}

ABSTRACT. It is shown that when the kernel of a homogeneous Volterra integral equation is singular, it is possible for there to be a continuous spectrum of eigenvalues.

1. Introduction. Consider the Volterra integral equation

$$
\phi(x)=f(x)+\lambda \int_{0}^{x} K(x, y) \phi(y) d y .
$$

It is well known [1] that, if $K$ is continuous or weakly singular, the power series expression in $\lambda$ for the resolvent is convergent for all $\lambda$, and that, consequently, the homogeneous Volterra equation

$$
\phi(x)=\lambda \int_{0}^{x} K(x, y) \phi(y) d y
$$

does not possess any eigenvalues. What does not seem to be well known, [2] however, is that there can be, under certain conditions, a continuous eigenvalue spectrum when the kernel is singular.

This can be shown by a very simple example. From the result

$$
x^{n}=n \int_{0}^{x} y^{n-1} d y \quad \cdot(\operatorname{Re}(n)>0)
$$

it follows that a solution of the Volterra type integral equation

$$
\phi(x)=\lambda \int_{0}^{x} x^{-1} \exp \{x-y\} \phi(y) d y
$$

with the singular kernel $x^{-1} \exp \{x-y\}$ is

$$
\phi_{n}(x)=\exp (x) x^{n-1} \quad(\operatorname{Re}(n)>0)
$$

which has associated with it the eigenvalue $n$. There is thus a continuous spectrum of eigenvalues for the integral equation (4). An alternative

Received by the editors February 16, 1973.

AMS (MOS) subject classifications (1970). Primary 45C05, 45D05, $45 E 99$. 
representation of (4) would be given by writing $x \phi(x)=\psi(x)$, giving

$$
\psi(x)=\lambda \int_{0}^{x} y^{-1} \exp \{x-y\} \psi(y) d y .
$$

In this case the kernel $y^{-1} \exp \{x-y\}$ is still singular.

2. Analysis. Consider now the Volterra type integral equation

$$
a(x) \phi(x)=\lambda \int_{0}^{x} m(x, y) \phi(y) d y
$$

the kernel of which is $\{a(x)\}^{-1} m(x, y)$. Suppose now that $m(x, y)$ is of the form $m(x-y)$. This will simplify the analysis somewhat, but does not affect the ideas involved. Suppose that

$$
\begin{aligned}
& a(x)=\sum_{s=0}^{\infty} a_{s} x^{s+\alpha}, \\
& m(x)=\sum_{s=0}^{\infty} m_{s} x^{s+\mu},
\end{aligned}
$$

and look for a solution of the form

$$
\phi(x)=\sum_{s=0}^{\infty} \phi_{s} x^{s+\xi}
$$

$\alpha$ and $\mu$ are known, $\xi$ is to be determined. Obviously $a_{0}, m_{0}, \phi_{0}$ are nonzero.

Equation (7) can now be rewritten in the form

$$
\sum_{s=0}^{\infty} a_{s} x^{s+\alpha} \sum_{s=0}^{\infty} \phi_{t} x^{t+\xi}=\lambda \int_{0}^{x} \sum_{s=0}^{\infty} m_{s}(x-y)^{s+\mu} \sum_{t=0}^{\infty} \phi_{t} x^{t+\xi} d x
$$

which simplifies further to

$$
\begin{aligned}
\sum_{s=0}^{\infty} x^{s+t+\alpha+\xi} & \sum_{t=0}^{s} a_{s-t} \phi_{t} \\
& =\lambda \sum_{s=0}^{\infty} \sum_{t=0}^{\infty} x^{s+t+\mu+\xi+1} m_{s} \phi_{t} \int_{0}^{1}(1-Z)^{s+\mu} Z^{t+\xi} d Z \\
& =\lambda \sum_{s=0}^{\infty} \sum_{t=0}^{\infty} x^{s+t+\mu+\xi+1} m_{s} \phi_{t} B(s+\mu+1, t+\xi+1) \\
& =\lambda \sum_{s=0}^{\infty} x^{s+\mu+\xi+1} \sum_{t=0}^{s} m_{s-t} \phi_{t} B(s-t+\mu+1, t+\xi+1) .
\end{aligned}
$$

It will be noted that for the integrals to converge, it is necessary that $\operatorname{Re}(\mu+1)$ and $\operatorname{Re}(\xi+1)$ should be positive. In order that the leading term of both series be the same, it follows that $\alpha+\xi=\mu+\xi+1$, which implies that $\alpha=\mu+1$. (Although apparently a possible solution would be given by 
$\alpha-\mu-1$ being equal to an integer $N$, it can easily be seen that this would imply $\phi_{s}$ vanishing for $s<N$ and so is irrelevant.) Equation (7) now assumes the form

$$
\sum_{s=0}^{\infty} x^{s} \sum_{t=0}^{s} a_{s-t} \phi_{t}=\lambda \sum_{s=0}^{\infty} x^{s} \sum_{t=0}^{s} m_{s-t} \phi_{t} B(s-t+\mu+1, t+\xi+1)
$$

as $x^{\alpha+\xi}$ cancels. It follows from equation (12) that

$$
\sum_{t=0}^{s}\left(a_{s-t}-\lambda m_{s-t} B(s-t+\mu+1, t+\xi+1)\right) \phi_{t}=0, \quad s \geqq 0 .
$$

The eigenvalue is determined by taking the case $s=0$. This gives

$$
a_{0}-\lambda m_{0} B(\mu+1, \xi+1)=0
$$

or

$$
\lambda=a_{0} /\left\{m_{0} B(\mu+1, \xi+1)\right\} .
$$

Now $\xi$ is not defined, save by the convergency condition referred to previously, and so it follows that the spectrum of $\lambda$ as given by equation (15) is continuous. The set of equations (13) can, using (15), be rewritten as

$$
\sum_{t=0}^{s}\left[a_{s-t}-\frac{m_{s-t} a_{0} B(s-t+\mu+1, t+\xi+1)}{m_{0} B(\mu+1, \xi+1)}\right] \phi_{t}=0 .
$$

The set of equations (16) gives recurrence relations for $\phi_{s}$ in terms of $\phi_{s-1}, \cdots, \phi_{0}$. (It can easily be verified that the coefficient of $\phi_{n}$ does not vanish in (16) when $s=n$.) It will be noted that the $\phi_{s}$ are in fact functions of $\xi$. Thus the sum of the series

$$
\sum_{s=0}^{\infty} \phi_{s}(\xi) x^{s+\xi}
$$

where the $\phi_{s}(\xi)$ are defined by equation (16) is, if it converges, and if $\operatorname{Re}(\xi+1)$ is positive, an eigenfunction for the integral equation (7), the corresponding eigenvalue being given by equation (15).

\section{REFERENCES}

1. V. I. Smirnov, Course in higher mathematics. Vol. IV, 3rd ed., GITTL, Moscow, 1953; English transl., Pergamon Press, Oxford; Addison-Wesley, Reading, Mass., 1964, p. 136. MR 31 \#1333.

2. The author has consulted 17 books on integral equations, but has found no reference to this.

School of Mathematics and Computer Science, University College of North Wales, Bangor, CaERnarfonshire, Wales 